\title{
Misty role of postoperative adjuvant therapy in resected invasive intraductal papillary mucinous neoplasm of the pancreas: A multicenter external validation
}

\author{
Munseok $\mathrm{CHOl}^{1}$, Chang Moo KANG ${ }^{* 1}$, Sung Hoon $\mathrm{CHOI}^{2}$ \\ 'Department of Surgery, Yonsei University College of Medicine, Seoul, Korea \\ ${ }^{2}$ Department of Surgery, CHA Bundang Medical Center, Seongnam, Korea
}

Introduction: Adjuvant therapy is beneficial in prolonging survival in patients with pancreatic ductal adenocarcinoma (PDAC). However, no clear guidelines are available on the oncologic effect of adjuvant therapy in resected invasive intraductal papillary mucinous neoplasms (inv-IPMN).

Methods: In total, 551 patients with PDAC and 67 patients with inv-IPMN of the pancreas were reviewed. For external validation, 46 patients with inv-IPMN from six other Korean institutions were enrolled. Stage-matched survival analysis was conducted.

Results: Mean follow-up durations in the inv-IPMN and PDAC groups were 54.13 and 30.82 months, respectively. The overall 5-year survival was significantly better in the resected inv-IPMN group than in the PDAC group in the overall stage-matched analysis $(p<$ 0.001). Comparison of disease-free survival by stage showed a significant difference in overall stage between inv-IPMN and PDAC ( $p<$ $0.001)$. Among patients with stage II disease $(p=0.012)$ and lymph node metastasis $(p=0.004)$, adjuvant therapy had a negative effect on overall survival (OS) in patients with resected inv-IPMN. Multicenter external validation did not show a better OS in the adjuvant therapy group. On multivariable analysis, only perineural invasion (PNI) was identified as an adverse prognostic factor in resected inv-IPMN (hazard ratio 4.844 ; 95\% confidence interval 1.696 to $13.838, p=0.003$ ).

Conclusions: Inv-IPMN has a more indolent course than PDAC. PNI was the strongest prognostic factor in the resected inv-IPMN group. Current strategy of adjuvant therapy may not improve the OS in patients with resected inv-IPMN. Further investigations on potential role of adjuvant therapy in inv-IPMN is mandatory. 